\title{
Scheme to Measure One-way Delay Variation with Detection and Removal of Clock Skew
}

\author{
Makoto Aoki, Eiji Oki, and Roberto Rojas-Cessa
}

\begin{abstract}
One-Way Delay Variation $(O W D V)$ has become increasingly of interest to evaluate network state and service quality, especially for real-time and streaming services such as VoIP and video. Measurement of these parameters needs to be performed with the layout infrastructure. Many schemes for OWD measurements require clock synchronization at the source and destination through Global-Positioning System (GPS) or the Network Time Protocol (NTP). In clock-synchronized approaches, the accuracy of the measurement of $O W D V$ depends on the achieved accuracy of clock synchronization. GPS provides high-accuracy clock synchronization. However, the deployment of GPS on legacy network equipment might be slow and costly. This paper proposes a method for measuring $O W D V$ without recurring to clock synchronization. However, clock skew may affect the measurement of $O W D V$. The proposed approach is based on the measurement of Inter-Packet Delay $(I P D)$ and Accumulated $O W D V(A O W D V)$. This paper shows the performance of the proposed scheme via simulation and through experimentation in a VoIP network. The presented simulation and experimental results indicate that clock skew can be efficiently measured and removed and that $O W D V$ can be measured without requiring clock synchronization.
\end{abstract}

Index Terms-delay measurement, clock skew, clock rate adjustment, one-way delay, inter-packet delay.

\section{INTRODUCTION}

Real-time applications over the Internet, such as Voice-overIP (VoIP) and video streaming services, use One-Way Delay Variation $(O W D V)$ information to monitor and maintain Quality of Service (QoS) or quality perception by the user. $O W D$ is define as the delay that a data packet suffers in the trip from source to destination. If the source and destination use the same clock, this is equivalent to the difference between the arrival time at the destination minus the departure time at the source. $O W D V$ is define as the difference of $O W D$ s experienced by two packets of the same length [3]. According to [3], the definitio of $O W D V$ between any two packets $a$ and $b$, where $a \neq b$, is the difference of their $O W D$ s.

$$
O W D V(a, b)=O W D(a)-O W D(b) .
$$

However, the source and destination use different clocks in the general case. Therefore, a large effort has been made in the recent years to provide clock synchronization between remote hosts.

Makoto Aoki is with Cyber Creative Institute, Tokyo, Japan, Email: aoki@cybersoken.com. He is also a Visiting Researcher at The University of Electro-Communications, Tokyo, Japan, Email:aoki@cybersoken.com

Eiji Oki is with The University of Electro-Communications, Tokyo, Japan, Email:oki@uec.ac.jp.

Roberto Rojas-Cessa is with the Dept. of Electrical and Computer Engineering, New Jersey Institute of Technology, Newark, NJ 07102, USA.

Email: rojas@njit.edu.
To describe clock synchronization, the following definition are used in this paper. Clock $\mathrm{A}, C_{A}$ has time $t_{A}$ at the same time that Clock B, $C_{B}$, indicates $t_{B}$, where $t_{A}$ and $t_{B}$ are the indicated times or time stamps at the same time. The offset time is $O_{A, B}=t_{A}-t_{B}$. The clocks $\mathrm{A}$ and $\mathrm{B}$ advance at rate $r_{A}=\frac{d\left(t_{A}\right)}{d t}$ and $r_{B}={ }_{d t}^{d\left(t_{B}\right)}$, respectively. The clock drift is the rate of change of the clock rate. However, for simplicity, this clock drift is considered to be zero. Then the clock skew using $C_{A}$ as reference is define as $S_{A}={ }_{r_{A}}$. In similar way the clock skew referenced at $C_{B}$ is $S_{B}=\frac{r_{A}}{r_{B}}$.

Clock synchronization is the adjustment of time on the local clock in relation to the other end's clock to make the measurements in reference to a single time scale, using as the reference the clock at the source, destination, or any other. In other words, clock synchronization is the adjustment of the clocks' offsets.

There are two broad groups of measurements approaches, active and passive. Active measurements inject probing packets into the network, specificall between source and destination hosts, with the purpose to measure the network parameter of interest [6], [7], [8]. In several of the existing activemeasurement schemes, Global Positioning System (GPS), Network Time Protocol (NTP) [4], or Precision Time Protocol (PTP), also known as IEEE 1588 standard [5], are used with the objective to perform clock synchronization. However, the introduction of GPS increases equipment costs. Furthermore, it is difficul to be deployed on legacy equipment.

NTP and PTP require to measure Round-Trip Time (RTT) to perform clock synchronization, and even more restrictive, they require each way (from source to destination and from destination to source) has equal $O W D$ to function. In general, each way has a different $O W D$ unless a network has been engineered with this feature in mind, and it is difficul to expect that from a network such as the Internet.

An active measurement scheme to measure $O W D$ that does not require clock synchronization was proposed [9]. This scheme needs to select cyclic-paths properly to calculate each one-way delay. However, it is based on the measurement of round-trip delays.

A passive measurement scheme uses the fl wing traffi in the network, usually user traffic to perform the measurement process. Passive measurement is attractive because it does not need to inject additional traffi to the network and because measurement is directly performed on the user traffi (the effect that the user actually experiences). However, the accuracy of this approach depends on the fl wing traffi at a given time, which is beyond the control of the network operator. A passive-measurement scheme to measure $O W D$ was recently proposed [10]. This scheme, yet passive, it is based on GPS and NTP for clock synchronization. Another scheme for 
measurement of $O W D$ was proposed [11]. However, it is based on the assumption that PTP achieves clock synchronization.

Recently, a scheme to measure $O W D$ clock skew removal was proposed [15]. This scheme uses the real time protocol (RTP) [1] and the Real-Time Control Protocol (RTCP) [2] to create fi ed length and fi ed inter-packet delay, IPD, to perform $O W D$ measurements. IPD is the time between two consecutive packet departures at the source and measured at the destination. This approach is based on a simple model to estimate clock skew, showing a different perspective from the existing complex approaches to the estimation of clock skew, where for example, linear programming is used [16]. However, the efficien y of clock skew removal depends on experiencing low queuing delays during the measurement. However, this depends on the user traffi rather than in the measurement method. The case where the network remains with long queuing delays is not considered in the scheme.

This paper proposes a scheme to measure $O W D V$ without requiring to clock synchronization between the source and destination hosts. The proposed approach, measured IPDs and the combined and accumulated $O W D V$, called $A O W D V$, which is the sum of continuing IPDs. However, as clock skew is included in the IPD measurements, this paper uses a mechanism to estimate and remove clock skew using $A O W D V$. $O W D V$ is obtained from $A O W D V$ s after clock skew is removed. In this process, the proposed method estimates the clock-rate difference between the source and the destination. Evaluation of the proposed approach is presented with both simulation and analysis of real network data. The evaluation results show that the proposed method can effectively estimate $O W D V$ as derived from $I P D$.

The remainder of this paper is organized as follows. Section II introduces the proposed mechanism to measure $O W D V$. This section also describes a methodology detection and adjustment of clock skew on the performed measurements. Section III presents a simulation study of the proposed $O W D V$ scheme. Section IV presents the experimental results of $O W D V$ evaluations on a VoIP network. Section V presents our conclusions.

\section{Scheme to MeAsure One-WAy Delay VARiation}

The proposed mechanism is based on the measurement of the inter-packet delay IPD at the destination host. The adopted method, uses the smallest experienced IPD, called $D_{\text {min }}$, to evaluate the contribution of every new IPD measurement as packets are sent from source to destination. For the evaluation of the clock skew, the source explicitly sends packets, where the dispatching time between consecutive packets at the source host is conveyed to the destination. The packets have fi ed length to comply with the $O W D V$ definitio [3]. Every packet has an identificatio number. For the description of the proposed mechanism, $i$ is the firs packet transmitted from the source to perform measurement. Then, $I P D(i+1)$ is measured between packets $i$ and $i+1$. Figure 1 shows an example of the delay times between packets $i$ and $i+1$ in the transmission of multiple packets. In this example, packet $i$ departs at time $T(i)$ and packet $i+1$ at time $T(i+1)$. The arrival times of packets $i$ and $i+1$ are then $R(i)$ and $R(i+1)$. For simplicity, it assumes that packets are transmitted in order of sequence. If out-of-order packets were received at the destination, these packets would be discarded. In that case, $T S$ and $I P D$ between $i+j$ and $i+k(k>j+1)$ of correctly received packets are measured.

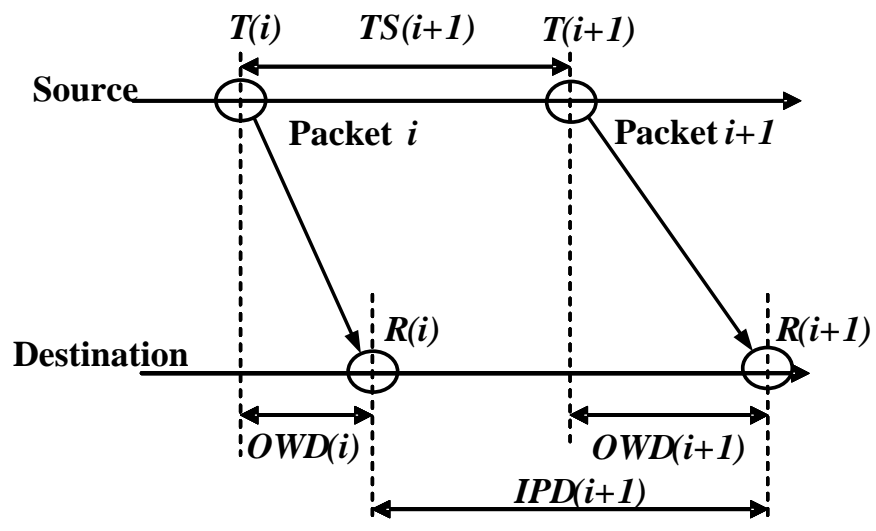

Fig. 1. Variables to measure $O W D V$ based on $I P D$.

The inter-packet delay at the source, $T S(i+1)$, is define as

$$
T S(i+1)=T(i+1)-T(i),
$$

This value is set by the source. The inter-packet delay measured at the destination, $\operatorname{IPD}(i+1)$, is:

$$
I P D(i+1)=R(i+1)-R(i)
$$

This value is $T S$ plus the variable $O W D$ that a packet undergoes, and it is measured in reference to the clock at the destination. The value of $O W D$ would have several parameters, or $O W D=t_{p}+t_{t}+t_{q}$, where $t_{p}, t_{t}$, and $t_{q}$ are the total propagation, transmission, and queuing delays accumulated in the path from source to destination hosts. These variables are susceptible to variations caused by paths or traffi variations during the measurement period. This paper, rather than focusing on the measurement of these specifi parameters, is centered on the measurement of the variations of $O W D, O W D V$.

The measurement of $O W D V$ is based on the measurement of $I P D$. As shown in Figure $1, \operatorname{IPD}(i+1)$ is measured between packets $i$ and $i+1$, which in this case are consecutive packets, and the minimum IPD experienced among all those measured, denoted as $D_{\text {min }}$, including that of packet $i+1$. The consideration of consecutive packets is not required as long as $T S$ between two packets is conveyed to the destination host (there are several options for the implementation of this requirement [11], however, they are left out of the scope of this paper).

Here, $D_{\min }$ uses as reference value the smallest $O W D$ collected during the measurement period. Figure 2 describes the relationship of $O W D V$ and $D_{\min }$.

The experienced $O W D V$ by packet $i+1$ in reference to $D_{\text {min }}$ can be re-written as

$$
O W D V(i+1)=O W D(i+1)-D_{\min }
$$

On the other hand, the inter-packet delay at the source can be described in function of $I P D$ :

$$
T S(i+1)+O W D(i+1)=O W D(i)+I P D(i+1)
$$




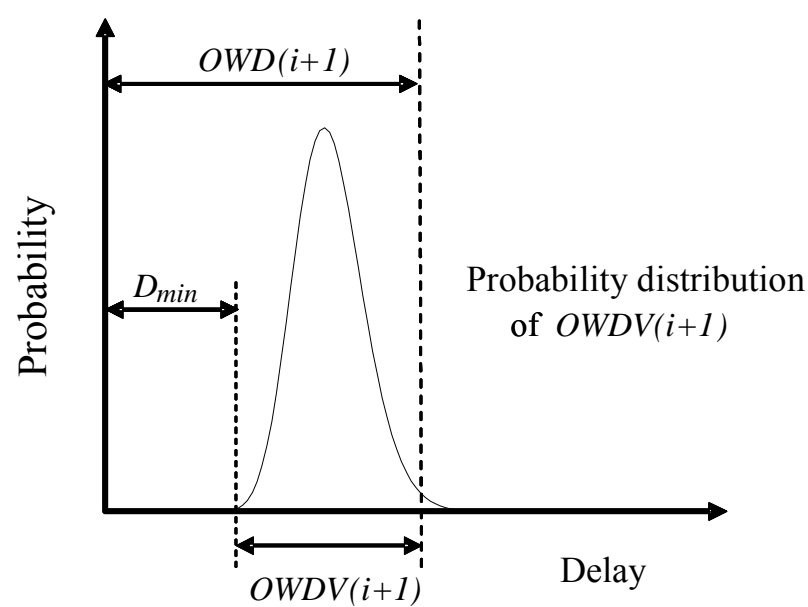

Fig. 2. Relationship of $D_{\min }$ and $O W D V(i+1)$.

Using (5) and for a long measured period, $O W D(i+n)$ of the $O W D$ for the last received packet $n$, can be expressed as

$$
O W D(i+n)=\sum_{j=1}^{n}(I P D(i+j)-T S(i+j))+O W D(i)
$$

where the sum of collected $(I P D(i+j)-T S(i+j)$ is called $A O W D V$ or:

$$
A O W D V(i+n)=\sum_{j=1}^{n}(I P D(i+j)-T S(i+j)) .
$$

Using (7) $O W D(i+n)$ can be described as:

$$
O W D(i+n)=A O W D V(i+n)+O W D(i) .
$$

$D_{\min }$ in (4) can be expressed in terms of (6) as

$$
\begin{gathered}
D_{\min }=\min \{O W D(i+j)\} \quad j=1,2 \ldots n \\
=\min \{A O W D V(i+1), \ldots, \\
A O W D V(i+n)\}+O W D(i)
\end{gathered}
$$

where $\min \left\{x_{j}\right\} j=1,2 \ldots n$ is define as a function of selecting a minimum value in a set of $\left\{x_{1}, x_{2}, \ldots x_{n}\right\}$.

Using (8) and (9), $O W D V$ for packet $n$ can be expressed as:

$$
\begin{aligned}
& O W D V(i+n)=A O W D V(i+n) \\
& -\min \{A O W D V(i+1), \ldots, A O W D V(i+n)\}
\end{aligned}
$$

This is, $O W D V$ uses the smallest $D_{\min }$ experienced during the measurement time, independently of the firs experience $O W D$, or $O W D(i)$.

\section{A. Removal of Clock Skew}

The removal (or also called adjustment) of the clock skew from the $O W D V$ measurements are needed. The adjustment needs to be done in reference to the destination clock $C d$. Measurements of $T S$ are made with the source clock, so they include information about the clock rate of $C s$. Since

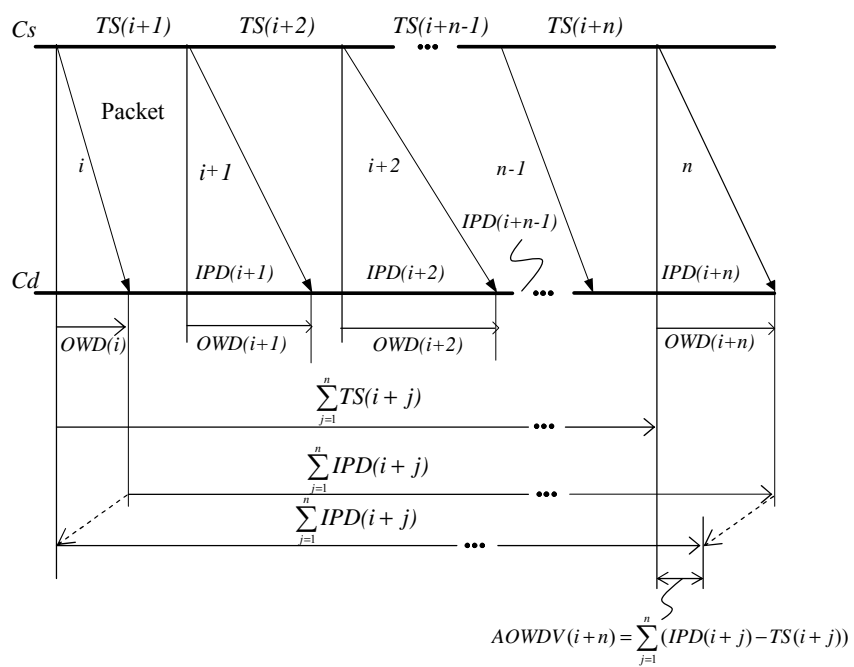

Fig. 3. Example of data collected for clock synchronization.

the information is the difference of the departure times, clock synchronization is not needed.

For this, the accumulated $O W D V$ as in (7) per-packet basis is considered. Figure 3 shows a general description of how $A O W D V$ is collected. $A O W D V$ contains clock skew information (considering the rate of change of clock skew equal to zero) that can be observed when this parameter is plotted against time $(C d)$. However, no every $A O W D V$ value provides useful information. For this, only the smallest values of $A O W D V, \min \{A O W D V\}$, including negative values are used. The selection of $\min \{A O W D V\}$ is independent of the difference of the smallest value in the complete set of collected measurement (i.e., $n$ ), which can make it dependable of user traffic Instead, information of the rate of small values collected is used to divide the measurement time in windows whose size is determined by the rate of small values $r(l)$ collected in $n$ samples under normalized traffi load $(l)$, which uses the link or network capacity as reference value. This is

$$
r(l)=\frac{N s(l)}{n}
$$

where $N s(l)$ is the number of small $A O W D V$ values collected in $n$ packets at load $l$. The window size is them given in number of packets.

\section{Simulation Study of the Proposed Scheme}

Simulation of a VoIP environment was performed using the network simulator ns2 [17]. The network traffi (CBR) simulated a VoIP traffic using 240-byte packets every 20 ms, using $96 \mathrm{kbps}$ of bandwidth. In addition, there are three independent $\mathrm{fl}$ ws of cross traffics modeled as variable bit rate (VBR) traffic of which both packet lengths and intervals have exponential distributions. The traffi is assumed to be data traffi using TCP/IP with an average packet length of 700 bytes. The clock rate difference between source and destination is set to 1000 parts per million (ppm), or $1 \mathrm{~ms}$. Figure 4 shows the network model used in the simulation. In this model, a VoIP transmission is performed between a source host and a destination host. The traffi for this transmission 
is modeled as CBR traffic Data are captured as a network operator would collect them in an actual network. Only IPD values are collected. Each packet carries a timestamp, using the local clock (i.e., Cs). With these data, $O W D V$ and $A O W D V$ are obtained, whoever, including clock skew information.

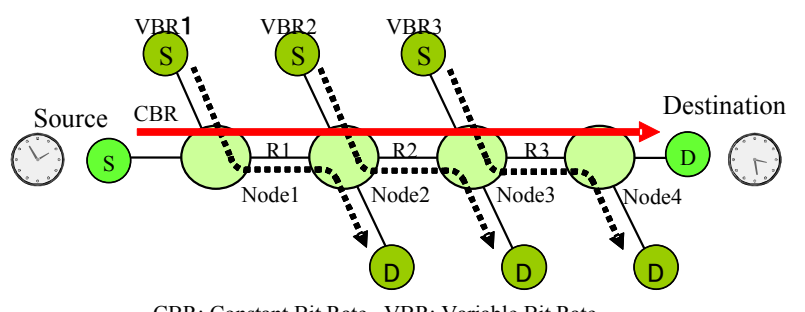

CBR: Constant Bit Rate VBR: Variable Bit Rate

$\boldsymbol{\cdots} \rightarrow$ Cross-traffic (VBR) $\longrightarrow$ : Observed-traffic (CBR)

Fig. 4. Network simulation model for $O W D V$ estimation.

The network load (define as the traffi load on the tight link, which is the link with the smallest capacity) was set at $80 \%$. Figure 5 shows the collected $A O W D V$ data, calculated from the collected $T S$ and $I P D$ values. $A O W D V$ is plotted vs. the arrival times as clocked by $C d$. In addition to the variability in the $A O W D V$ observed created by the queuing delays of the cross traffic the figur shows that there is a steady offset increase in the $A O W D V$ measured. This offset increase is created by the clock skew.

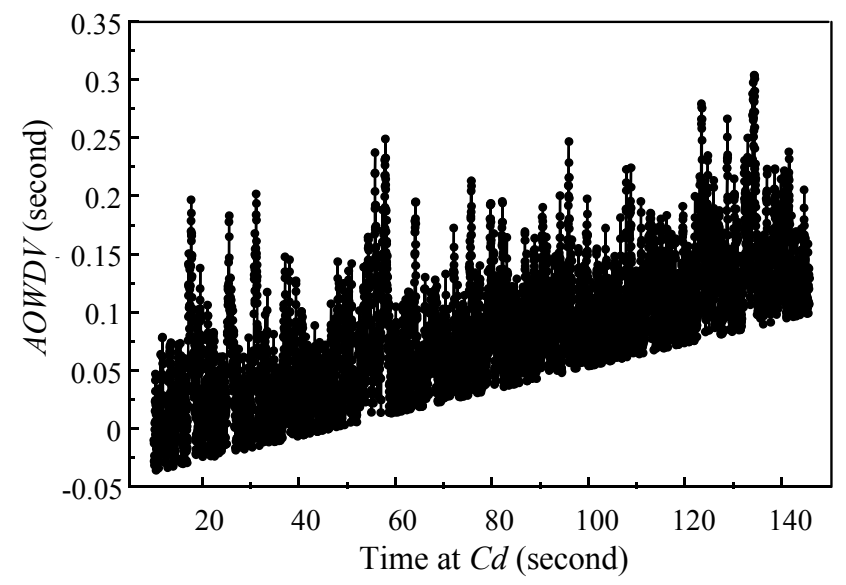

Fig. 5. $I P D$ with offset produced by clock skew between $C s$ and $C d$.

To remove the clock skew, the smaller values of $A O W D V$ are collected, as define in Section II-A. Different from [15], where an acceptable error $\epsilon$ from the minimum experienced delay is used to determine the selection of delays for the calculation of the clock skew, the approach adopted in this paper segments the simulation time into windows, and from each window, the lower values are collected. The windows selected here have a length of 100 packets, or 2 seconds (as CBR traffi is used).

Figure 6 shows the minimum values of $A O W D V$ collected. These values shows similar linear increasing offset to that observed in Figure 5. The advantage of this method is that even if queuing delay remains constant, the collectible $A O W D V$ values can be used for the calculation of the clock skew. In the error approach, if the queuing delay is constant but larger than $\epsilon$, no values can be collected, requiring longer time and lower queuing delays, which are out of the control of network operators as the queuing delays are caused by the cross traffic

The clock skew was estimated using linear regression, and the resulting is $1037.39 \mathrm{e}-06$. This means that the calculation the clock skew has an error of $3.7 \%$ (in reference to the 1000 e-06 in the experiment setup). After the skew is calculated, it is removed from the measured $O W D V$. This is the actual $O W D V$

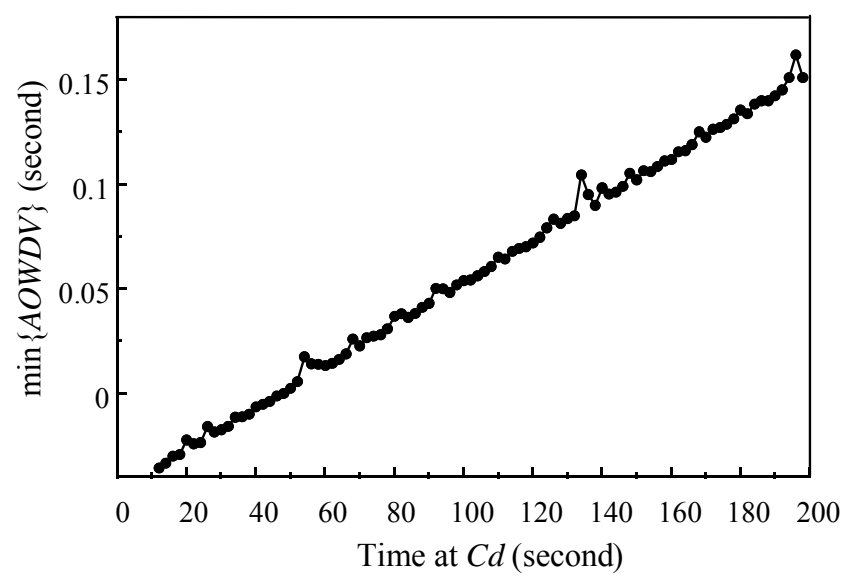

Fig. 6. Minimum $A O W D V$ values used for clock skew estimation.

Figure 7 shows $O W D V$ of this experiment, where the offset created by the clock skew is removed. As the figur shows, the image shows similar distribution to that shown in Figure 5 , so the information is kept.

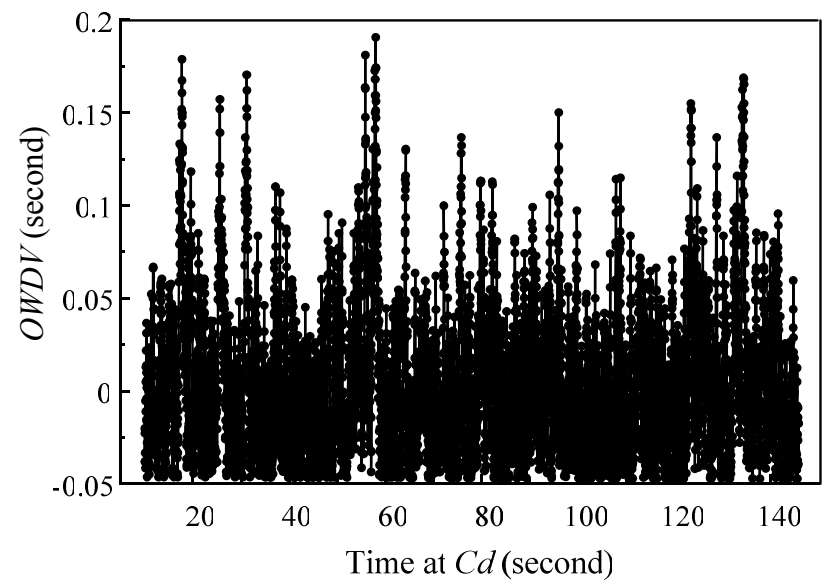

Fig. 7. $O W D V$ with clock skew removed.

\section{Experimental Test of the Proposed Scheme}

$O W D$ measurements were experimented in a practical fiel environment. The used system offers private VoIP services to around 20,000 IP phones over major cities in Japan. The networks consist of various network subsystems such as private core networks, public VoIP networks, Internet, ADSL, 
Wireless LAN (IEEE 802.11). The network systems, including IP phones, are run without clock synchronization.

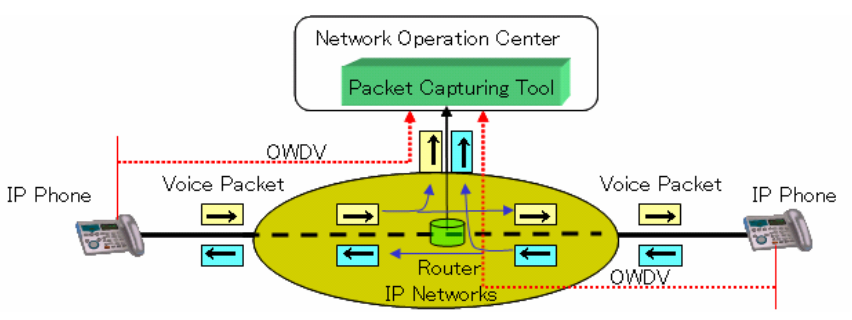

Fig. 8. Network model for experimental measurement of $O W D V$.

VoIP quality metrics such as packet loss and jitter except one-way delay (variation) are monitored at the Network Operation Center (NOC). Figure 8 shows the experimental system used to monitor the transmitted voice packets. The voice packets on the monitoring paths are copied and transferred to packet capturing tools installed at the NOC. The packet capturing tools record the information of proper RTP packets including transmission (timestamp) and reception times. Information of VoIP calls is stored in the used packet capturing tools to estimate $O W D$. The source of this information is indicated with red dotted lines in the figure

Figure 9 shows the estimated $A O W D V$ based on sampled data at the NOC. The $A O W D$ shows an apparent constant offset, indicating that clocks skew is small.

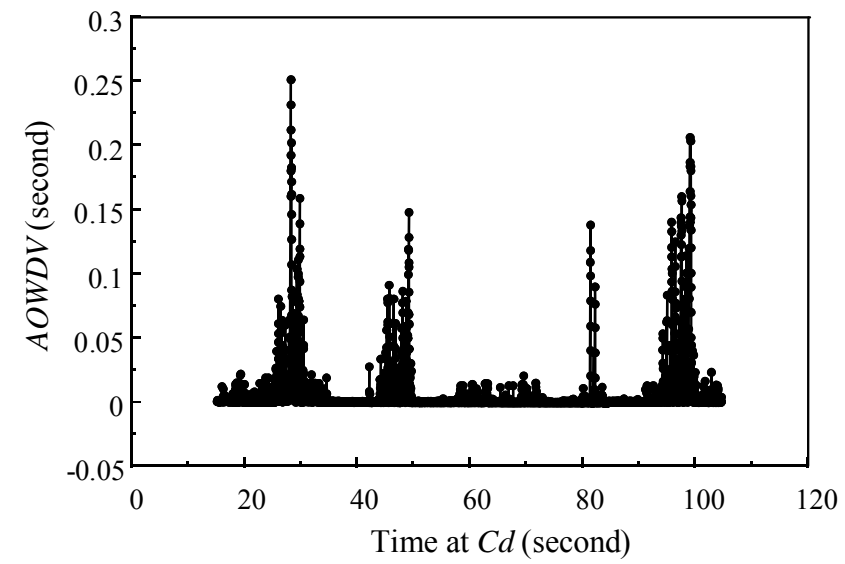

Fig. 9. $A O W D V$ of values collected in the experimental VoIP network.

The collected minimum values of $A O W D V$ are shown in Figure 10. The values, due to the duration of the collection process, shows a variation that could indicate the magnitude of the clock skew, when in fact the largest values are the most representative, as $C d$ has slightly smaller rate than $C s$.

The VoIP calls related to examples are characterized by having large jitters among the captured calls. The figur shows that the clock skew between the source and the destination are almost zero and the values of this clock skew varied between 3 and $17 \mathrm{ppm}$ at the collection time.

Figure 11 shows the $O W D V$ of the collected values after clock skew removal. In this case, the small clock skew looks removable by a simple process and most data shows similar

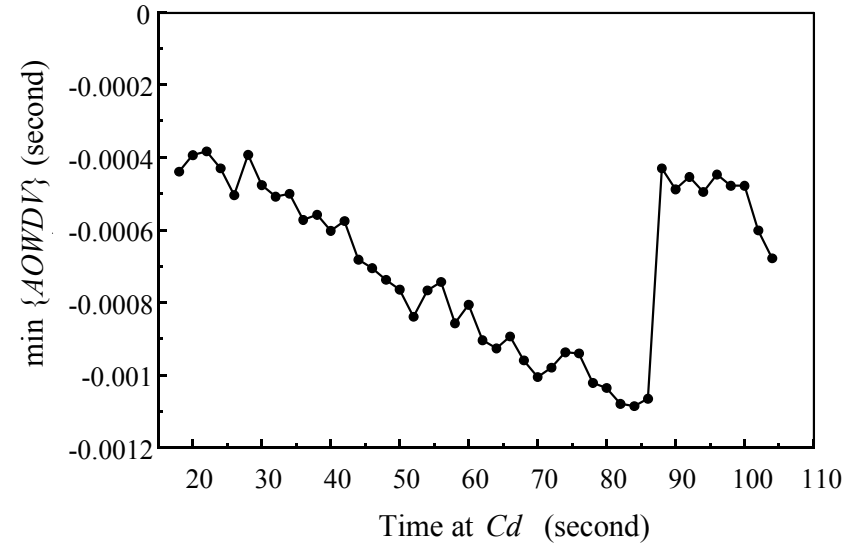

Fig. 10. Minimum $A O W D V$ values in the the firs experiment in the experimental VoIP network.

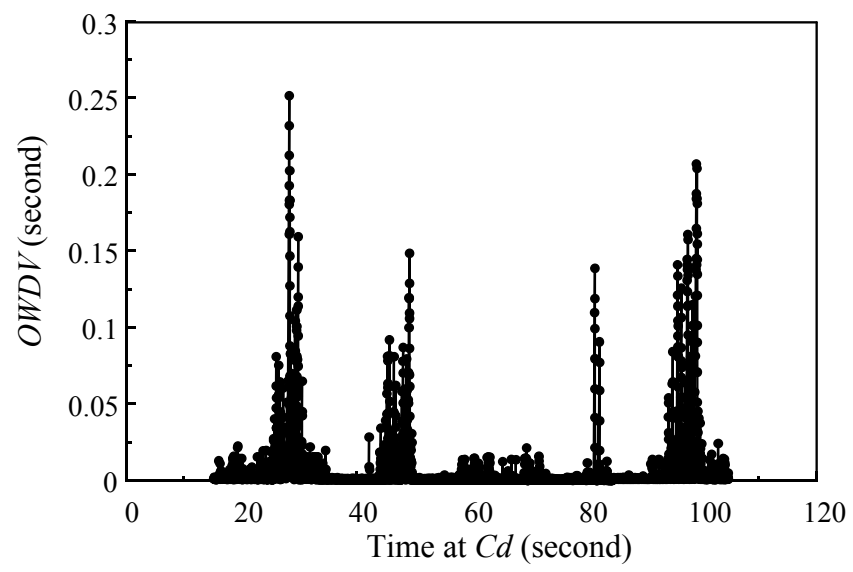

Fig. 11. $O W D V$ of the firs experiment in the experimental VoIP network.

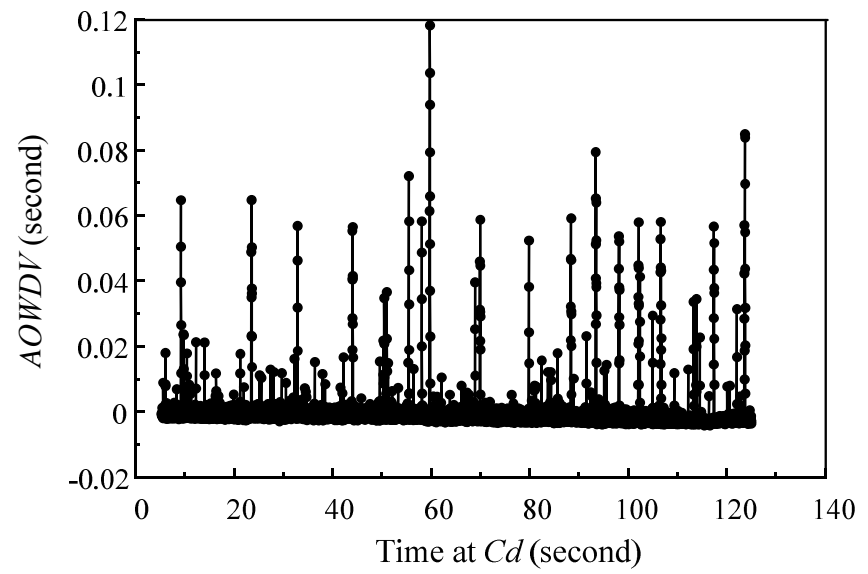

Fig. 12. $A O W D V$ values in the second experiment in the experimental VoIP network.

trend. However, linear regression is used for this purpose, applied on the minimum values.

In a second experiment, the new collected data shows a smaller variation of the offset values indicated the clock skew. Figure 12 shows the $A O W D V$ values of the this experiment. 


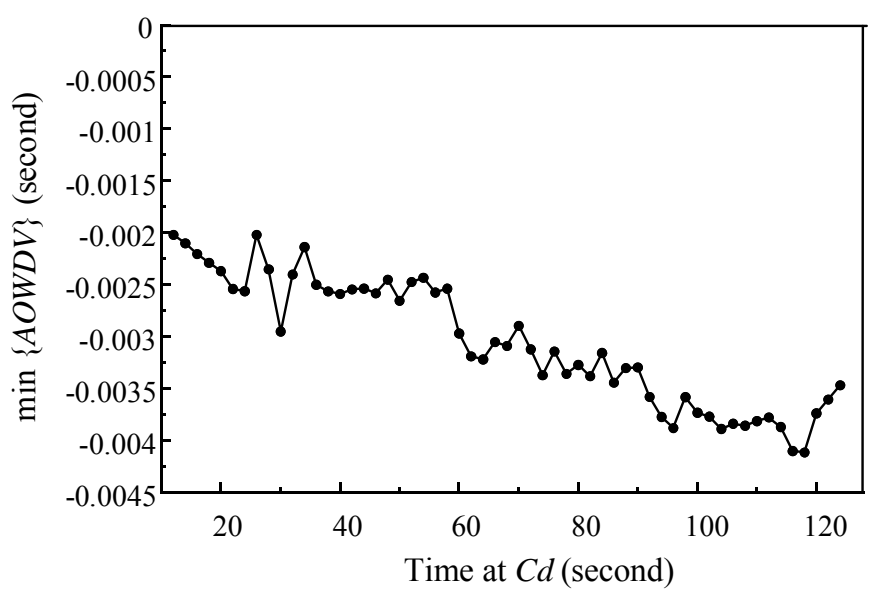

Fig. 13. Minimum $A O W D V$ values of the second experiment in the experimental VoIP network.

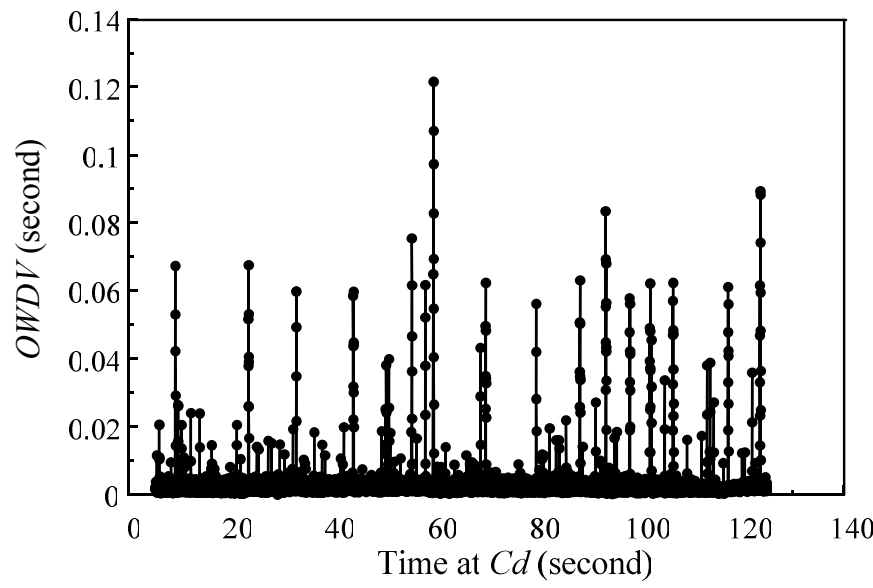

Fig. 14. $O W D V$ of the second experiment in the experimental VoIP network.

The offset values have a small slope, indicating a small clock skew in this practical network.

Figure 13 shows the minimum values of $O W D V$ used to detect the clock skew. Figure 14 shows the $O W D V$ of this experiment with the clock skew removed after using linear regression among the minimum $A O W D V$ values. The figur shows the $O W D V$, which shows similarities to the $A O W D V$ shown in Figure 12. As shown in the $O W D V$, the clock skew is effectively removed without affecting the network information.

\section{CONCLUSIONS}

One-way delay variation $(O W D V)$ experienced by traveling packets between source and destination host can be easily measured if clock synchronization is performed between these two hosts. Clock synchronization has been proved difficul with means other than the use of GPS as time adjustment protocols require symmetrical paths for round trips measurements. However, as legacy equipment may need total replacement for the deployment of GPS, this replacement may be slow or expensive. As an alternative to use additional resources, this paper presents a scheme for measuring one-way delay variation $(O W D V)$ that does not require clock synchronization. The method depends on the clock skew adjustment. This paper presents the dependability of $O W D V$ on the clock skew. The proposed measurement scheme is based on the measurement of inter-packet delays (IPDs), which is the difference of the arrival times between consecutive packets. The IPD values are closely related to the $O W D V$ but however, they are affected by the clock skew between the source and destination clocks. This paper shows a method for the estimation and detection of clock skew to enable the measurement of $O W D V$. Different from other existing methods, the method to estimate the clock skew is based on the accumulated values of $O W D V$ or $A O W D V$. The use of $A O W D V$ speeds up the collection of samples as it does not discard useful data as in error-based methods.

The proposed measurement scheme was tested with computer simulation and on an experimental VoIP network. The simulation and experimental results show that the clock skew can be effectively removed and that the $O W D V$ values can be efficientl obtained. The error for clock skew estimation achieved was equal to or smaller than $3.7 \%$. The proposed method can by network operators to detect large jitters or $O W D V$ and to make network adjustments.

\section{REFERENCES}

[1] H. Schulzrinne, S. Casner, R. Frederick, V. Jacobson, "RTP: A Transport Protocol for Real-Time Applications," RFC 3550, July 2003.

[2] C. Huitema, "Real Time Control Protocol (RTCP) attribute in Session Description Protocol (SDP)," RFC3605, October 2003.

[3] C. Demichelis and P. Chimento, "IP Packet Delay Variation Metric for IP Performance Metrics (IPPM)," RFC 3393, Nov. 2002

[4] D. L. Mills, "Network Time Protocol (Version 3) Specification Implementation and Analysis," RFC 1305, 1992.

[5] Caleb Gordon, "Introduction to IEEE 1588 and Transparent Clocks," White Paper, Tekron, 2009

[6] Y. Shavit, et al., "Large scale Internet Queueing Delay Tomography," Proc. IEEE INFOCOM 2006, 3 Pages, Barcelona, Catalunya, SPAIN, April 23-29, 2006.

[7] T. Iwama, A. Kaneko, A. Machizawa, H. Toriyama, Real-Time Measurement of One-Way Delay in the Internet Environment, Proc. 2004 Communications Society Conference of IEICE, B-16-1, 1 Page, September 2004

[8] U. Hofmann et al., "One-Way-Delay Measurements with CM Toolset," Proc. IEEE Int'l Conference on Performance, Computing, and Communications (IPCCC 2000), 7 Pages, Phoenix, Arizona, Feb. 20-22, 2000.

[9] O. Gurewitz, et al., "Estimating One-Way Delays From Cyclic-Path Delay Measurements," INFOCOM 2001, 7 Pages Anchorage, Alaska, USA, April 22-26, 2001.

[10] T. Zseby, et al., "Passive One-Way-Delay Measurement and Data Export," Proc. The International Workshop on Inter-domain Performance and Simulation, 6 Pages, Salzburg, Austria, Feb. 20-21, 2003.

[11] M. Cola, et al., "Covert Channel for One-Way Delay Measurements," Proc. International Conference on Computer Communications and Networks (ICCCN) 2009, 6 Pages, San Francisco, CA, USA, Aug. 3-6, 2009.

[12] http://www.wireshark.org

[13] http://www.empirix.com/products/hammer_xms_service_assurance.asp

[14] http://www.isi.edu/nsnam/ns/

[15] B. Ngamwongwattana and R. Thompson, "Measuring One-Way Delay of VoIP Packets without Clock Synchronization," Proc. IEEE International Instrumentation and Measurement Technology Conference (IMTC) 2009, 4 pages, Singapore, May 5-7, 2009.

[16] S.B. Moon, P. Skelly, and D. Towsley, "Estimation and Removal of Clock Skew from Network Delay Measurements," IEEE INFOCOM '99, Vol.1, 8 pages, New York, NY, USA, March 21-25, 1999.

[17] ns2 network simulator, http://www.isi.edu/nsnam/ns, Available Online, July 2007. 